\title{
Evaluación de la velocidad de transferencia de datos entre diferentes compañías telefónicas
}

\section{Evaluation of data speed transfer between different telephone companies}

\author{
Moreno Irian ${ }^{1}$, Mudarra Marian ${ }^{1}$,Ureña Yennifer ${ }^{l}$, Vega Guadalupe ${ }^{1}$, Maritza Cedeño $^{2}$ \\ ${ }^{1}$ Licenciatura en Ingeniería Industrial - Centro Regional de Azuero - Universidad Tecnológica de Panamá \\ ${ }^{2}$ Grupo de Investigación de Azuero en el Área Empresarial
}

\begin{abstract}
Resumen En este artículo se muestra la aplicación de un diseño cuadrado latino para evaluar si existe diferencia significativa en la velocidad de transferencia de datos entre las diferentes compañías telefónicas de Panamá. El diseño propuesto define dos factores de bloque que se controlan en el experimento, estos factores son: tipo de celular y la hora del día. Para asegurar la independencia de los datos se aleatorizaron los tratamientos dentro de los bloques. La variable dependiente, velocidad de transferencia de datos, fue medida a través de una aplicación denominada "SpeedTest". Mediante análisis de varianza se encontró que la hipótesis inicial fue rechazada al 5\% de significancia, por lo que existen suficientes evidencias como para concluir que hay diferencia en la velocidad de transferencia de datos entre las empresas telefónicas en Panamá.
\end{abstract}

Palabras clave Velocidad, transferencia de datos, diseño de experimento, teléfono móvil.

\begin{abstract}
This article describes the application of a Latin square design to assess whether there is a significant difference in the rate of data transfer between different telephone companies in Panama. In the proposed design two block factors are controlled: cell type and time of day. It should be noted that to ensure the independence of the data treatments are randomized within blocks. The data transfer rate was measured through an application called " SpeedTest ". By analysis of variance it was found that the initial hypothesis was rejected at $5 \%$ significance so that there is sufficient evidence to conclude that there is difference in data transfer speed of telephone companies in Panama.
\end{abstract}

Keywords Speed, data transfer, design experiment, mobile phone.

* Corresponding author: yennifer.urena@utp.ac.pa

\section{Introducción}

La Ingeniería Industrial tiene entre sus principales propósitos el diseño, mejora e instalación de procesos, aplicando conocimientos a la solución de problemas. En este campo, la experimentación es una herramienta fundamental que ayuda a recolectar y analizar datos para una correcta toma de decisiones con respecto a los fenómenos que pueden presentarse en dichos procesos.

Un experimento diseñado es una serie de pruebas en las cuales se inducen cambios deliberados en las variables de entrada de un proceso o sistema de manera que sea posible observar e identificar las causas de los cambios en la respuesta de salida [1].

La presente investigación tiene como base la experimentación sobre uno de los más utilizados dispositivos electrónicos a nivel mundial, el teléfono celular. De los diferentes tipos de diseños de experimentos resultó de mayor adecuación a nuestro estudio el Diseño Cuadrado Latino, el cual es un diseño que se utiliza para controlar dos fuentes de variabilidad durante el experimento. En otras palabras, este permite analizar sistemáticamente por bloques en dos direcciones. En este diseño los renglones y las columnas representan en realidad dos restricciones de aleatorización [1].

En este experimento se requiere la aleatorización de los tratamientos, las columnas y los renglones, entendiéndose por aleatorización como la técnica utilizada para reducir la incertidumbre de los resultados, aumentando la confianza de la investigación [2]. Luego de realizada la aleatorización se puede proceder de manera confiable a la realización del experimento.

Los principales objetivos de esta investigación son:

1. Diseñar un experimento que permita la medición de la velocidad de transferencia de datos (carga) entre las diferentes telefónicas de Panamá.

2. Verificar si existe o no diferencia significativa entre los resultados del experimento. 
En la realización del experimento se siguieron los pasos del diseño de experimentos, los cuales son: [3]

1. Identificación y planteamiento del problema

2. Elección de los factores, niveles y riesgo

3. Selección de la variable de respuesta

4. Elección del diseño experimental

5. Realización del experimento (materiales, métodos)

6. Análisis de datos

7. Conclusiones.

\section{Planteamiento del problema}

Con el objeto de evaluar si la velocidad de transferencia de datos (velocidad de carga), difiere significativamente según la compañía proveedora de datos móviles, aplicamos un diseño cuadrado latino con cuatro tratamientos (servicio de datos de las cuatro compañías telefónicas de Panamá); seleccionamos cuatro períodos de tiempo, asignándolos a la columna y cuatro modelos de celular de la marca Samsung, asignándolos a los renglones.

\section{Diseño experimental}

A continuación, se describen los elementos principales que se han tomado en cuenta en el diseño experimental.

\subsection{Elección de los factores, niveles y riesgo}

Cuando se quiere realizar un experimento se debe tener el conocimiento de todas las variables que puedan intervenir en la realización de dicho experimento. En nuestro caso las variables que pueden afectar la medición de la transferencia de datos son: el tipo de compañía telefónica, el modelo de celular, la hora del día en la que se realiza el experimento, el uso que se le esté dando a la red internet, la cantidad de dispositivos móviles conectados y la cobertura de la señal, entre otros.

De los factores mencionados se seleccionan como tratamientos las diferentes compañías telefónicas y consideramos que se pueden controlar dos de estos factores, los cuales serán utilizados como bloque en nuestro experimento. Ellos son, el modelo de celular y la hora del día en la cual se realiza el experimento. El modelo de celular afecta el experimento debido a que a pesar de que se trata de la misma marca (SAMSUNG) la velocidad de transferencia de datos de cada celular depende del tipo de procesador que tenga en celular, la memoria RAM y el espacio que queda libre en el celular. Para nuestro experimento se eligieron cutro modelos diferentes de celulares de la marca Samsung. La hora del día en la que se realiza el experimento influye debido a que la variable en estudio depende de la cantidad de personas conectadas a la red en diferentes horas del día. Se seleccionaron cuatro horas de la mañana para la realización de experimento. Los demás factores son variables que no se pueden controlar, pero que afectarán por igual la velocidad de transferencia de datos durante el experimento.

Para el caso de los tratamientos se escogieron cuatro diferentes compañías telefónicas, representadas estas por las diferentes tarjetas SIM (Módulo de identificación del suscriptor) de cada una de ellas, por lo tanto, es un experimento de un factor con cuatro niveles.

Con relación al nivel de riesgo para este experimento se planteó un 5\% de significancia.

\subsection{Elección de la variable de respuesta}

La variable de respuesta en nuestro estudio es la velocidad de transferencia de datos (carga) la cual será medida con una aplicación de Play Store denominada Speed Test, la cual medirá la velocidad de los dispositivos móviles, sometidos a los diferentes tratamientos para transferir datos en mega bit por segundo, Mbps.

\subsection{Elección del diseño experimental}

Para la realización de este experimento se escogió un diseño cuadrado latino (doble bloqueo), debido a que este diseño nos permitió bloquear los dos factores que pudieron influir en el experimento.

La ecuación (1) presenta el modelo matemático [5] que corresponde a este diseño:

$$
y_{i j l}=\mu+T_{j}+\rho_{j}+\delta_{l}+E_{i j l}
$$

En donde:

$y_{i j l}=$ Observación del tratamiento $i$, en el nivel $j$ del factor renglón y en el nivel 1 del factor columna.

$\mu=$ Efecto medio verdadero.

$E_{i j l}=$ Error atribuible a la observación.

La hipótesis que se desea comprobar al final del experimento es:

$\mathrm{H}_{0}=$ No existe diferencia significativa en la velocidad de transferencia de datos entre las diferentes compañías telefónicas.

\section{Materiales y métodos}

En los dos apartados siguientes se describen los materiales y la metodología utilizada en la investigación.

\subsection{Materiales}

Se utilizaron cuatro modelos diferentes de dispositivos móviles o celulares marca Samsung y cuatro tarjetas SIM con datos móviles activados (una por cada compañía telefónica).

La medición de la velocidad de transferencia de datos se realizó con la aplicación SpeedTest. Esta es una herramienta especializada disponible de manera "online" en computadoras y como "aplicación" en celulares.

Aunque SpeedTest mide otros elementos, nos enfocamos en los resultados de la velocidad de transferencia de datos (velocidad de carga), la cual se mide en megabits por segundo (Mbps). 


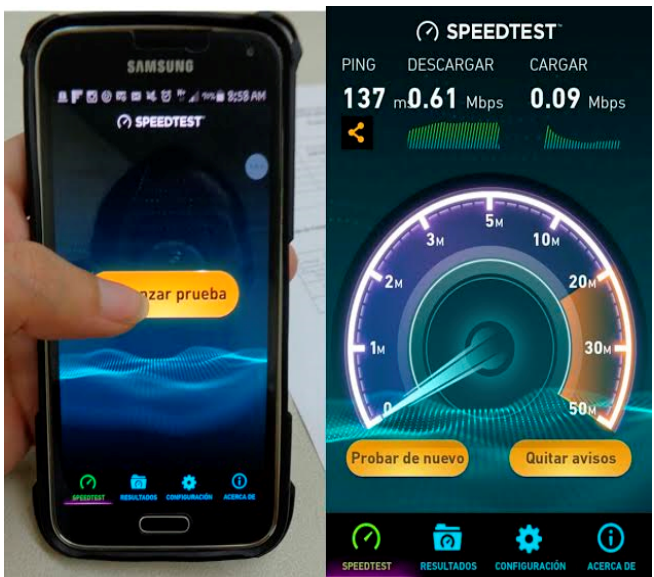

Figura 1. "SpeedTest" herramienta de medición.

\subsection{Metodología}

El experimento inicia con el proceso de aleatorización. La adaptación del experimento al diseño parte de un cuadrado latino estándar del tamaño requerido (4x4), se aleatorizan todas las columnas del cuadrado elegido y seguidamente todas las filas del cuadrado encontrado, ambas aleatorizaciones se realizaron con ayuda de una calculadora. Esta aleatorización permitirá la independencia de los datos haciendo la investigación más confiable. Igualmente, se asegura, como se recomienda en este diseño, que no se repitan los tratamientos en cada fila y en cada columna. En la tabla 1 se muestra el arreglo final de los tratamientos.

Tabla 1. Codificación y aleatorización

\begin{tabular}{|c|c|c|c|c|}
\hline & \multicolumn{4}{|c|}{ Hora del Día } \\
\hline $\begin{array}{c}\text { Tipo de } \\
\text { celular }\end{array}$ & 09:00 a. m. & 10:00 a. m. & $11: 00$ a. m. & 12:00 m. d. \\
\hline $\begin{array}{c}\text { Grand } \\
\text { Prime }\end{array}$ & C & A & B & D \\
\hline $\begin{array}{c}\text { Grand Neo } \\
\text { Plus }\end{array}$ & D & B & C & A \\
\hline Modelo $\mathrm{S}_{4}$ & A & C & D & B \\
\hline Modelo $\mathrm{s}_{5}$ & B & D & A & C \\
\hline
\end{tabular}

La velocidad de conexión a Internet es calculada por SpeedTest de la siguiente manera, primero, se realiza la descarga de un archivo de $500 \mathrm{~KB}$ desde el servidor hasta el celular donde se realiza la prueba. En la figura 2 se muestra la ejecución del experimento. En cada réplica del experimento, se calcula el tiempo en que se tarda cada celular, con su respectiva tarjeta SIM, en completar la descarga. Una vez que tenemos estos datos, la aplicación nos muestra la velocidad de conexión a Internet [4].

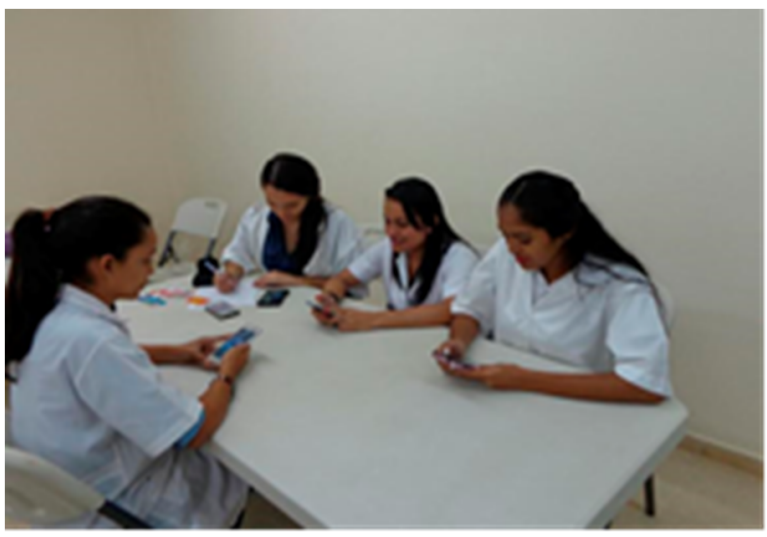

Figura 2. Ejecución del experimento.

\section{Análisis y discusión de los resultados}

Con la aplicación SpeedTest se procede a la recolección de los datos y los resultados de las mediciones se muestran en la tabla 2.

Tabla 2. Velocidad de transferencia de datos en Mbps según el tipo de celular y la hora del día

\begin{tabular}{|c|c|c|c|c|}
\hline \multirow{2}{*}{$\begin{array}{c}\text { Tipo de } \\
\text { Celular }\end{array}$} & \multicolumn{4}{|c|}{ Hora del Día } \\
\cline { 2 - 5 } & $09: 00$ a. m. & $10: 00$ a. m. & $11: 00$ a. m. & $12: 00$ p. m. \\
\hline $\begin{array}{c}\text { Grand } \\
\text { Prime }\end{array}$ & $0.36 \mathrm{C}$ & $1.14 \mathrm{~A}$ & $0.27 \mathrm{~B}$ & $3.32 \mathrm{D}$ \\
\hline $\begin{array}{c}\text { Grand } \\
\text { Neo } \\
\text { PLUS }\end{array}$ & $3.17 \mathrm{D}$ & $0.09 \mathrm{~B}$ & $0.54 \mathrm{C}$ & $1.14 \mathrm{~A}$ \\
\hline $\mathbf{S}_{\mathbf{4}}$ & $1.89 \mathrm{~A}$ & $0.11 \mathrm{C}$ & $1.10 \mathrm{D}$ & $0.27 \mathrm{~B}$ \\
\hline $\mathbf{S}_{\mathbf{5}}$ & $0.33 \mathrm{~B}$ & $1.98 \mathrm{D}$ & $1.32 \mathrm{~A}$ & $0.71 \mathrm{C}$ \\
\hline
\end{tabular}

Una vez recolectados los datos procedimos a insertarlos en Minitab para obtener el análisis de varianza y de esta manera poder concluir acerca del experimento y validar la hipótesis.

La tabla 3 muestra el resultado para las tres fuentes de variación analizadas.

Tabla 3. Análisis de varianza

\begin{tabular}{|c|c|c|c|c|c|}
\hline Fuente & GL & SC Sec & $\begin{array}{c}\text { SC } \\
\text { ajust. }\end{array}$ & $\begin{array}{c}\text { MC } \\
\text { ajust. }\end{array}$ & $\mathbf{F}$ \\
\hline $\begin{array}{l}\text { Tratamiento } \\
\text { (Telefónica) }\end{array}$ & 3 & 11.73 & 11.73 & 3.91 & 11.18 \\
\hline $\begin{array}{c}\text { Renglones (tipo } \\
\text { de celular) }\end{array}$ & 3 & 0.46 & 0.46 & 0.15 & 0.44 \\
\hline $\begin{array}{c}\text { Columnas (hora } \\
\text { del día) }\end{array}$ & 3 & 1.36 & 1.36 & 0.45 & 1.29 \\
\hline Error & 6 & 2.10 & 2.10 & 0.35 & \\
\hline Total & 15 & 15.64 & & & \\
\hline
\end{tabular}




\subsection{Resultado 1}

Para los tratamientos (Tipos de empresas telefónicas), Minitab nos muestra un valor $\mathrm{F}$ de 11.18 y el valor $\mathrm{F}$ tabulada es decir $\mathrm{F}(5 \%, 3,6)$ es 4.76 , lo que indica que el valor $\mathrm{F}$ calculado cae en el área de rechazo de $\mathrm{H}_{0}$, como se puede observar en la figura 3.

Lo anterior significa que existe suficiente evidencia como para concluir que hay diferencia en la velocidad de transferencia de datos entre empresas telefónicas, al 5\% de significancia.

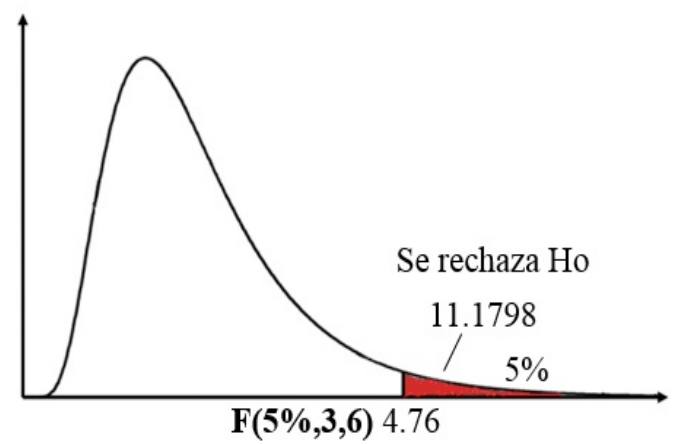

Figura 3. Gráfico de Fisher para el tratamiento.

\subsection{Resultado 2}

De los datos obtenidos también podemos hacer un análisis de la velocidad promedio de trasferencia de datos vs las diferentes telefónicas y como muestra la figura 4 , al comparar el comportamiento gráfico de los promedios nos damos cuenta que en efecto existe una diferencia en las velocidades de trasferencia de datos.

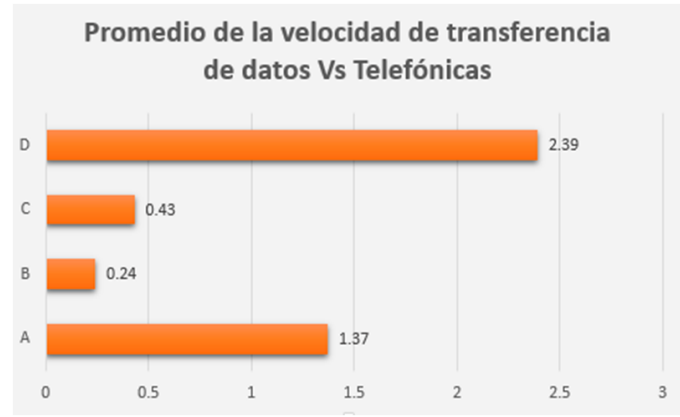

Figura 4. Gráfico de Promedios de los tratamientos.

\section{Conclusiones}

$\mathrm{Al}$ culminar el experimento se puede concluir, que sí existe diferencia significativa en la velocidad de transferencia de datos entre las diferentes telefónicas, debido a que la hipótesis inicial fue rechazada, a un 5\% de significancia.

Cabe destacar la importancia de la elección del diseño experimental adecuado debido a que la influencia de diversos factores pudo haber afectado la realización del experimento. Sin embargo, al introducir los datos en Minitab se pudo analizar que los bloques, en este caso, el tipo de celular y la hora del día, no tuvieron un efecto en la realización del experimento por lo cual, para futuros estudios se puede realizar el experimento mediante un diseño completo al azar.

\section{RECONOCIMIENTO}

A la Ingeniera Maritza Cedeño de Moreno por su apoyo y motivación para desarrollar nuestra investigación experimental. También agradecemos a las personas que permitieron la utilización de sus teléfonos móviles para llevar a cabo el experimento.

\section{REFERENCIAS}

[1] Douglas C. Montgomery. Diseño y Análisis de experimentos. Segunda edición. Limusa, México, D.F, 2011.

[2] Antonio N Hurtado, Federico C. Domínguez. Probabilidad y estadística para Ingeniería. Primera edición México: McGraw Hill. 2010.

[3] Luis H Rojas Cárdenas, Lucio Rojas Cortés. "Exploración al diseño experimental" Ciencia e ingeniería Neogranadina. Junio 2000.

[4] Online available: Cablevisión 2014. http://Portal.Speedtest.Monterrey.

[5] Humberto G. Pulido, Román De la V. Salazar. (2008). Análisis y Diseño de Experimentos. Segunda edición. México: McGraw Hill. 2008.

\section{RIC}

